\title{
Detection of $\beta$-lactamase in Haemophilus influenzae Isolates by Double Disk Synergy Test
}

\author{
Harunur Rashid ${ }^{1 *}$ and Mahbubur Rahman ${ }^{2}$ \\ ${ }^{1}$ Institute of Oral Health Research and Department of Cell Biology, University of Alabama at Birmingham, USA \\ ${ }^{2} I C D D R, B$ : Centre for Health and Population Research, Bangladesh
}

\begin{abstract}
A total of 90 isolates of Haemophilus influenzae were included in this study. Among them $47.8 \%$ were resistant to ampicillin and amoxicillin. Of the 90 isolates 43 were positive for $\beta$-lactamase by cefinase test and the rest (47) were $\beta$-lactamase negative. Interestingly, all the $\beta$-lactamase positive isolates were ampicillin resistant. No isolate of $H$. influenzae was $\beta$-lactamase negative ampicillin resistant (BLNAR). All $\beta$-lactamasepositive ampicillin resistant (BLPAR) isolates were also positive for $\beta$-lactamase by double disk (Ampicillin and Amoxicillin/clavulanate) technique. And all $\beta$-lactamasenegative isolates were also negative double disk synergy test. Both the sensitivity and specificity of double disk synergy test were $100 \%$ compared to cefinase test. So, double disk synergy test can be used to detect $\beta$-latamase as an alternative of cefinase test by incorporating an augmentin (amoxicillin/clavulanic acid) disk during antimicrobial susceptibility testing by disk diffusion method.
\end{abstract}

Keywords: $\beta$-lactamase; BLNAR; BLPAR; $H$. influenza; Double disk synergy test

\section{Introduction}

Haemophilus influenzae is a major causative agent of community acquired respiratory tract infections (CARTIs), such as acute otitis media, bacterial rhinosinusitis, chronic bronchitis, pneumonia and meningitis. Approximately $0-30 \%$ of $H$. influenzae isolates (average $15 \%)$ are $\beta$-lactamase-positive $[1,2]$. Ampicillin/amoxycillin was the most effective treatment option for Haemophilus infection until the recent past. Resistance to ampicillin results from the production of a $\beta$-lactamase and alteration of the antibiotic target, penicillin-binding protein $[3,4] . \beta$-lactamase an enzyme which cleavage the $\beta$-lactam ring of ampicillin/amoxycillin antibiotic and make them ineffective in killing bacterial pathogen that produce $\beta$-lactamase. As a result the bacterial pathogens showed resistance to $\beta$-lactam antibiotic, such as ampicillin, amoxicillin, benzyle penicillin etc. Many bacterial pathogens such as $H$. influenzae, Staphylococcus aureus, Moraxella catarrhalis, etc. have been reported to produce $\beta$-lactamase. Treatment problems linked to the prevalence of $\beta$-lactam resistance are compounded by the frequency of combine resistance to many other antibiotics in addition to penicillin, such as chloramphenicol, cotrimoxazole, tetracycline etc [5]. Recent studies indicate the increasing trend of ampicillin resistance among $H$. influenzae isolates from the different part of the world [68]. For better management of invasive infections due to $\beta$ - lactamase mediated ampicillin (penicillin) resistant bacterial pathogens, it is important to detect $\beta$-lactamase enzyme accurately as well as cost effectively. We standardized a method for $\beta$-lactamase detections simultaneously during the testing of antimicrobial susceptibility by incorporating an additional disk augmentin (amoxicillin/clavulanic acid). The double disk synergy method is very effective in detection of $\beta$-lactamase producer and eliminated the additional cost for $\beta$ lactamase detection by cefinase disk test.

\section{Materials and Methods}

\section{Bacterial strains}

A total of 90 isolates of $H$. influenzae were included in this study. Of the 90 isolates 43 were ampicillin resistant and the rest were susceptible to ampicillin by disk diffusion and E-test technique.

\section{Antimicrobial susceptibility}

Antibiotic susceptibility was qualitatively determined by disk diffusion method according to Standard Clinical laboratory Institute (Previously National Committee for Clinical Laboratory Standard; NCCLS) guideline [9]. Minimum inhibitory concentration (MIC) was also determined E-test according to Jorgensen et al., $[10,11]$ technique using antimicrobial strips of ampicillin, amoxicillin, azithromycin, trimethoprim-sulfamethoxazole (TMP-SMX), ciprofloxacin, chloramphenicol, ceftriaxone, (AB Biodisk, Solna, Sweden). Haemophilus testing media (HTM) supplemented with X and V factor was used for susceptibility testing of $H$. influenzae. The plates were incubated in candle extinction jar $\left(10-15 \% \mathrm{CO}_{2}\right)$ at $37^{\circ} \mathrm{C}$ for $24 \mathrm{~h}$ prior to reading of the results. MICs were interpreted by using Standard Clinical laboratory Institute breakpoints. The MIC was defined as the lowest concentration of antibiotic that inhibited the visible growth. Isolates were considered multi- resistant if they had reduced susceptibility to at least three structurally unrelated antibiotics. $H$. influenzae ATCC 49247 strains was used for the quality control.

\section{$\beta$-Lactamase Detection Assay}

\section{Double disk synergy test (DDST)}

This test was performed on Haemophilus test medium (HTM) by using ampicillin and augmentin disk in addition to other antibiotic disks in the same antimicrobial susceptibility testing plate. The antibiotic disks containing bacterial culture plates were incubated at $370 \mathrm{C}$ for 20 22 hours. The plates were interpreted after incubation in three different

*Corresponding authors: Harunur Rashid, Institute of Oral Health Research and Department of Cell Biology, University of Alabama at Birmingham, USA, Tel: (205)996-5767; E-mail: hrashid07@gmail.com

Received October 29, 2015; Accepted November 16, 2015; Published November 23, 2015

Citation: Rashid H, Rahman M (2015) Detection of $\beta$-lactamase in Haemophilus influenzae Isolates by Double Disk Synergy Test. J Microb Biochem Technol 7: 417-418. doi:10.4172/1948-5948.1000248

Copyright: $\odot 2015$ Rashid H, et al. This is an open-access article distributed under the terms of the Creative Commons Attribution License, which permits unrestricted use, distribution, and reproduction in any medium, provided the original author and source are credited. 
ways. If the test isolates were susceptible to ampicillin and augmentin, no possibility of $\beta$-lactamase production, secondly if the isolate found to be resistant to ampicillin and susceptible to augmentin interpreted as $\beta$-lactamase positive ampicillin resistant (BLPAR) and finally if the test isolates showed resistance to both ampicillin and augmentin, were regarded as $\beta$ - lactamase negative ampicillin resistant (BLNAR).

\section{Cefinase test}

$\beta$-lactamase production in $H$. influenzae was determined using cefinase stick (nitrocefin) assay (Cefinase) (11). Quality control testing was conducted daily according to the manufacturer's instructions. Results were reported only when quality control results had been accepted.

\section{Results}

A total of 90 isolates (20 from CSF, 15 from blood and 55 from Nasopharyngeal swab) of Haemophilus influenzae were included in this study. Among the ninety isolates $47.8 \%$ were resistant to ampicillin or amoxicillin. Minimum inhibitory concentration of ampicillin resistant isolates was $4->256 \mu \mathrm{g} / \mathrm{ml}$ for ampicillin and 8->256 for amoxicillin (Table 1). Of the 90 isolates 43 (47.8\%) were positive for $\beta$-lactamase by cefinase test and the rest of 47 isolates were $\beta$-lactamase negative. All the $\beta$-lactamase positive isolates were ampicillin resistant. No $H$. influenzae isolate was $\beta$-lactamase negative ampicillin resistant (BLNAR). All $\beta$-lactamase positive ampicillin resistant (BLPAR) isolates were also positive by DDST. Consistently all $\beta$-lactamase negative isolates were also negative for $\beta$-lactamase by DDST. Both the sensitivity and specificity of DDST are $100 \%$ compared to cefinase test (Table 2).

\section{Discussion}

$H$. influenzae especially serotype $\mathrm{b}$ is one of the major cause of severe invasive infections, i. e., pneumonia, meningitis and septicemia among the extreme age group ( $<5$ years and $>65$ years). Ampicillin/ Amoxicillin were the most effective treatment option for Haemophilus infection until the recent past. Development of ampicillin resistance made the treatment difficult and led the physician to think about other options. Resistance to ampicillin results from the production of a $\beta$-lactamase and alternation of the penicillin-binding protein. Treatment problem linked to the prevalence of $\beta$-lactamase mediated resistance are compounded by the frequency of cross-resistance to many other antibiotics. So, it is very important to know the mechanism of ampicillin/amoxicillin resistance and the prevalence of $\beta$-lactamase mediated ampicillin resistance among the clinical isolates of $H$. influenza circulating in our community to develop an empirical treatment strategy.

\begin{tabular}{|c|c|c|c|c|c|}
\hline Antibiotics & \multicolumn{2}{|c|}{ MIC range $\left(\mu \mathrm{g} \mathrm{ml}^{-1}\right)$} & \multicolumn{3}{c|}{ Break point value $\left(\mu \mathrm{gl}^{-1}\right)$} \\
\hline & Susceptible & Resistant & $\mathrm{S}$ & $\mathrm{I}$ & $\mathrm{R}$ \\
\hline Ampicillin & $0.12-\leq 1$ & $\geq 4->256$ & $\leq 1$ & 2 & $\geq 4$ \\
\hline Amoxicillin & $0.25-\leq 2$ & $\geq 8->256$ & $\leq 2$ & 4 & $\geq 8$ \\
\hline
\end{tabular}

Table 1: Susceptibility and MIC result of colonized isolates of $H$. influenza.

\begin{tabular}{|c|c|c|c|}
\hline Cefinase stick test & \multicolumn{2}{|c|}{ Double disk synergy test } & \\
\hline & Positive $(n=43)$ & Negative $(n=47)$ & Total \\
\hline Positive & 43 & 0 & 43 \\
\hline Negative & 0 & 47 & 47 \\
\hline Total & 43 & 47 & 90 \\
\hline
\end{tabular}

Specificity $100 \%$ and sensitivity $100 \%$

Table 2: Comparison of two beta-lactamase testing methods for $H$. influenzae isolates.
Nitrocefin disk test was generally used for the detection of $\beta$-lactamase, which produce pink color by $\beta$-lactamase positive isolates and remain unchanged if the strain is $\beta$-lactamasenegative. Sometime $\beta$-lactamase positive isolates produce color not strong enough to detect visually and very confusing to conclude the result. But in case of double disk method the results could be obtained by the presence clear zone of inhibition without any chance to be confused. Moreover, this test could be accompanied with antimicrobial susceptibility testing without additional cost and efforts. This method allows detecting the ampicillin susceptibility as well as the mode ampicillin resistance simultaneously. So, the DDST can time, labor and additional cost for nitrocefin disk test. Sensitivity and specificity of double disk method is $100 \%$ compared to nitrocefin disk test. So, DDST can be used for the detection of $\beta$ - lactamase effectively fin $H$. influenzae as well as other $\beta$-lactamase producing bacteria such as Moraxella catarrhalis, Staphylococcus aureus, etc.

\section{References}

1. Felmingham D, Gruneberg RN (2000) The Alexander Project 1996-1997: latest susceptibility data from this international study of bacterial pathogens from community-acquired lower respiratory tract infections. J Antimicrob Chemother 45: 191-203.

2. Rahman M, Hossain S, Baqui AH, Shoma S, Rashid H, et al. (2008) Haemophilus influenzae type-b and non-b-type invasive diseases in urban children ( $<5$ years) of Bangladesh: Implications for therapy and vaccination. Journal of Infection 56: 191-196.

3. Markowitz SM (1980) Isolation of an ampicillin-resistant, non-blactamaseproducingstrain of Haemophilus influenzae. Antimicrob Agents Chemother 17: 80-83.

4. Mendelman PM, Chaffin DO, Stull TL, Rubens CE, Mack KD, et al. (1984) Characterization of non-b-lactamasemediated ampicillin resistance in Haemophilus influenzae. Antimicrob Agents Chemother 26: 235-244.

5. Talon D, Leroy J, Dupont MJ, Bertrand X, Mermet F, et al. (2000) Antibiotic susceptibility and genotypic characterization of Haemophilus influenzae strains isolated from nasopharyngeal specimens from children in day-care centers in eastern France. Clin Microbiol Infect 6: 519-524.

6. Shiro H, Sato $\mathrm{Y}$, Toyonaga $\mathrm{Y}$, Hanaki H, Sunakawa K, et al. (2015) Nationwide survey of the development of drug resistance in the pediatric field in 2000-2001, $2004,2007,2010$, and 2012: evaluation of the changes in drug sensitivity of Haemophilus influenzae and patients' background factors. J Infect Chemother 21: $247-256$

7. Zhu H, Wang A, Tong J, Yuan L, Gao W, et al. (2015) Nasopharyngeal carriage and antimicrobial susceptibility of Haemophilus influenzae among children younger than 5 years of age in Beijing, China. BMC Microbiol 15: 6 .

8. Marti S, Puig C, de la Campa AG, Tirado-Velez JM, Tubau F, et al. (2015) Identification of Haemophilus haemolyticus in clinical samples and characterization of their mechanisms of antimicrobial resistance. J Antimicrob Chemother.

9. National Committee for Clinical Laboratory Standards (NCCLS) (2000) NCCLS Performance Standard for Antimicrobial Susceptibility Testing, document M 1000-S13, Wayne, NCCLS

10. Jorgensen JH, Howell AW, Maher LA (1991) Quantitative antimicrobia susceptibility testing of Haemophilus influenzae and Streptococcus pneumoniae by using the E-test. J Clin Microbiol 29: 109-114.

11. O'Callaghan CH, Morris A, Kirby SM, Shingler SH (1972) Novel method for detecting $\beta$ - lactamase by using a chromogenic cephalosporin substrate. Antimicrob Agents Chemother 1: 283-288. 